\title{
A knowledge-based approach for design and modelling of high lift actuation systems
}

M Pfennig* and F Thielecke

Institute of Aircraft Systems Engineering, Hamburg University of Technology, Hamburg, Germany

The manuscript was received on 26 March 2010 and was accepted after revision for publication on 5 August 2010.

DOI: 10.1243/09544100JAERO857

\begin{abstract}
High lift actuation system design is a highly iterative process. Especially the preliminary design phase, which sets the course for the following design steps, is characterized by uncertain and changing data input. Despite the importance of this early design phase, a specialized and integrated computational support is not available. The objective of the research project WissBaSys is to offer knowledge-based design assistance focused on the preliminary design of high lift actuation systems. Moreover, information concerning a specific drive system architecture and component data can be used for an automated model generation leading to a significant time reduction and enhanced traceability. A capable approach for order reduction of shaft transmission models, as well as the concept for computer-aided modelling, is presented in this article.
\end{abstract}

Keywords: high lift actuation system, system design, modelling and simulation, flight control, systems engineering, knowledge-based systems

\section{INTRODUCTION}

The wing profile of modern transport aircraft is optimized for cruise speed in high altitudes. In contrast to the requirements in subsonic flight, significant lift augmentation is needed during the low-speed phases' take-off and approach. On commercial airplanes, high lift systems consist of moveable panels at the leading and trailing wing edge called slats and flaps, respectively. The extension of slats and flaps increases the effective wing area and camber. In consequence, higher lift and drag coefficients enable reduced take-off and landing speed.

Figure 1 shows the high lift surfaces and its actuation system. State-of-the-art high lift actuation systems predominantly consist of a central power drive unit (PDU) and a shaft transmission system providing mechanical synchronization of the spanwise distributed actuators [1]. These are either rotary actuators or ballscrew actuators with high gear ratios [2].

\footnotetext{
*Corresponding author: Institute of Aircraft Systems Engineering, Hamburg University of Technology, Nesspriel 5, Hamburg 21129, Germany.

email:malte.pfennig@tuhh.de
}

While the PDU features two redundant drive trains, each consisting of a brake and a hydraulic or electric motor unit, the mechanical transmission system has a single load path only. In order to prevent catastrophic impacts on the aircraft resulting from a single failure, safety devices are installed in the actuation system. A mechanical disconnect must not lead to uncontrollable flap or slat movement causing asymmetric flap setting or a sudden loss of the high lift function during a critical flight phase. Shaft brakes at the wing tip (wing tip brake, WTB) are active components that can stop and hold the shaft transmission in case of a mechanical disconnect. Alternatively, no-back devices, which are often installed in combination with ballscrew actuators, provide passive prevention from movement due to external air loads.

The actuation system can be divided into three major subsystems.

1. The PDU is installed in the centre fuselage. It supplies mechanical energy to operate the flap panels on each wing.

2. The shaft transmission system, which includes the actuators, converts the mechanical actuation from the power drive to a movement of the flap panels. In general, long and thin-walled shaft elements serve for power transmission. Angle gearboxes 


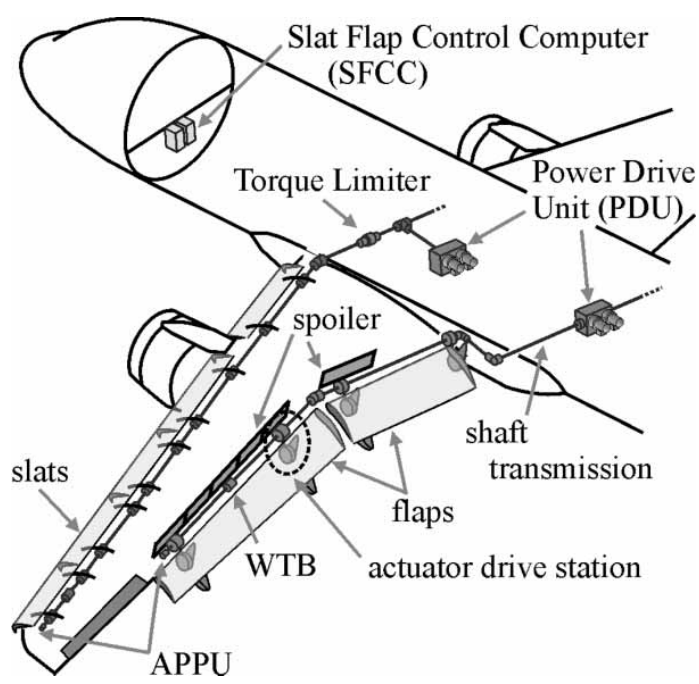

Fig. 1 Actuation of high lift systems [3]

enable directional changes in the shaft routing while universal joints, spline joints, and plunging joints compensate directional changes and length variations resulting from wing bending and temperature effects. Branch gears connect the actuator drive stations to the main transmission system.

3. The secondary flight control computer (SFCC) converts the pilot inputs to an actuation by commanding and controlling the PDU. Furthermore, system monitoring and failure detection are performed by the SFCC. Position sensor units at the wing tips (asymmetry position pickup unit, APPU) and at the PDU output provide signals for failure detection.

For testing the overall system behaviour and the interaction of these tree subsystems during nominal operation as well as in failure scenarios, simulation of the complex system behaviour is of major importance. However, establishing a detailed non-linear simulation model of the actuation system is complicated and time-consuming work. According to the computeraided approach presented in this article, the effort for model generation can be reduced significantly.

\section{DEVELOPMENT PROCESS}

The development process of aircraft systems can be divided into the following sections [4].

Requirements definition: Every system design process starts with the definition of the requirements. The high lift actuation system has to be able to operate at temperatures in the range of about -55 to $+70^{\circ} \mathrm{C}$. On these ambient conditions, the slat and flap panels have to be moved despite maximum opposing air loads within a specified time and accuracy. A fault-tolerant system is necessary and requirements regarding safety and availability targets have to be met. Furthermore synchronous panel movement has to be ensured. Some requirements like those concerning the installation space, for example, cannot be specified in early design phases. In contrast to the requirements, system weight, costs, and power consumption are the primary design criteria.

Conceptual or preliminary design: This phase of the design process starts with few and uncertain information. Basically, only the wing geometry, track positions, and actuator loads are roughly known at first. In the beginning, different concepts are analysed and rated in trade-off studies [5]. With advancing development time, one concept has to be chosen for further investigations and more detailed analysis. In general, reliable component data, which are necessary for an accurate prediction of the system behaviour, are not available. On the other hand, system interface loads for all relevant operating conditions are essential for specification not only of the system itself but also for its components. Hence, creating the system and component specification with respect to all requirements and design criteria requires high engineering skills and experience.

For most torque transmitting elements of high lift systems the maximum design loads are the primary design driver. Component weight, inertia, torsional stiffness, and other characteristics depend on the design loads. For determination of these loads, not only stationary operation has to be taken into account. Particularly inertia loads resulting from transient events, especially during failure scenarios, can mark the design load. For a reliable prediction of system loads with regard to non-linear effects, a complex simulation model is needed. However, required component data often change or might not be available for modelling. In consequence, complex and timeconsuming simulations are hardly available in the preliminary design phase.

Detail design: After the system design has been frozen and specified, subsystems and components are designed in detail. In this design phase, more requirements concerning the installation might be available. For detailed component design powerful computational assistance is available. Computeraided engineering (CAE) tools provide extensive help (e.g. computer-aided design (CAD) for generation of drawings or finite-element method (FEM) computations for strength analysis). In consequence, more reliable component data become available. For system engineering, these data are used to replace former presumptions. Repeating system analysis is necessary in order to recheck the specification and requirements. In parallel, a detailed system model can be established. Extensive and mature component data are now available as a basis for model definition.

Manufacturing: Finally the specified and designed system components are manufactured. Component data that were predicted from CAD can now be verified and fed back to the system engineer. 
Testing: After all, prototype components can be tested and calibrated. Furthermore, the complete system might be assembled from its subcomponents to a test rig. For system certification, extensive testing is necessary. Measurements can now provide final information that is needed for an accurate system simulation, like friction characteristics. A model representing the shaft transmission system is of major importance in this context. It allows for extensive hardware in the loop simulation and testing of the other subsystems. Thus, the simulation model can be validated to support the certification process.

In order to monitor the design goals and requirements, system analysis has to be repeated as soon as new and divergent system and component data become available. Early detection of discrepancies in system design is of major importance. The later the requirement violations are noticed, the higher the effort and cost for redesign [6]. Analysing the high lift actuation system and its requirements is a time-consuming task that can be automated once the necessary steps are defined [7].

The preliminary design is of significant importance for the entire process. The specification resulting from system analysis and trade-off studies is the basis for following development steps. Expert knowledge and experience is necessary to compensate missing or uncertain information. Moreover, design steps have to be evaluated repeatedly for predefined system architectures in order to perform concept studies. Even so, extensive and integrated computational assistance is not available. The computer-aided approach introduced in chapter 3 takes uncertainties into account explicitly and assists in finding a design solution. In consequence, development time and effort are reduced.

Another aspect regarding the design phases is the significance of system simulation. Owing to the effort and the required data for model generation considering non-linear effects, reliable simulation results become available not until the late detail design phase. One of the objectives of the computational design assistance for high lift actuation systems presented in the following is to transfer information concerning the system architecture and component data to a complex simulation model with minimum human effort. Thus, the main goal to provide extensive system simulation earlier in the design process might become available.

\section{COMPUTATIONAL DESIGN ASSISTANCE FOR HIGH LIFT ACTUATION SYSTEMS}

The previous analysis of the development process reveals that an integrated computational assistance and automation would be of high value especially in the preliminary design phase. However, a tool offering extensive support for the specialized design of high lift actuation systems is not available in early system design phases. The research project WissBaSys (German acronym for knowledge-based design of high lift actuation systems) [7] aims at this gap in the process chain. In order to provide assistance in the design process, a tool shall operate as a knowledge base and automate user-defined and time-consuming tasks.

System analysis is based on a specific architecture, as illustrated in Fig. 2 for the Airbus A330/340 flap actuation system exemplarily. In consequence, computations on system level have to take all components and their assembly into account.

Using different tools and static problem definitions for analysis requires high effort for adaptation in case of a changing architecture. In contrast, WissBaSys uses predefined component models that can be arranged and connected to a complex actuation system on a graphical user interface.

Figure 3 shows the graphical user interface of WissBaSys and its core elements. Generic models of typical components used in actuation systems are centralized in a component library. These generic component models contain parameterization according to the user's specification. Algebraic equations and inequalities describing the relation between component parameters are stored in the library models.

The elements contained in the library can be used to assemble system architectures. A database provides extensive data of existing high lift devices. If existing components shall be reused or served as a reference, appropriate datasets can be imported.

In order to enable high modelling flexibility the generic components in WissBaSys are equipped with ports, which represent mechanical interfaces. Thus,

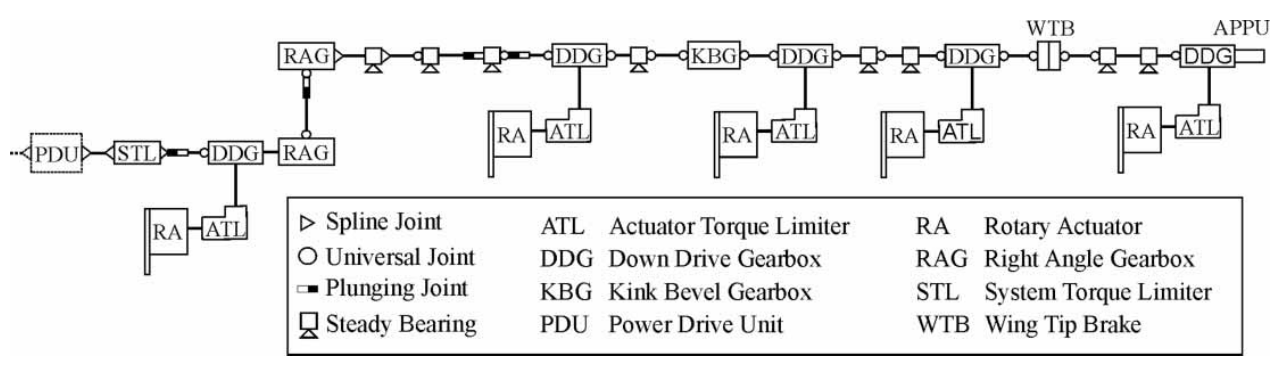

Fig. 2 Right wing of A330/340 flap actuation system [8] 


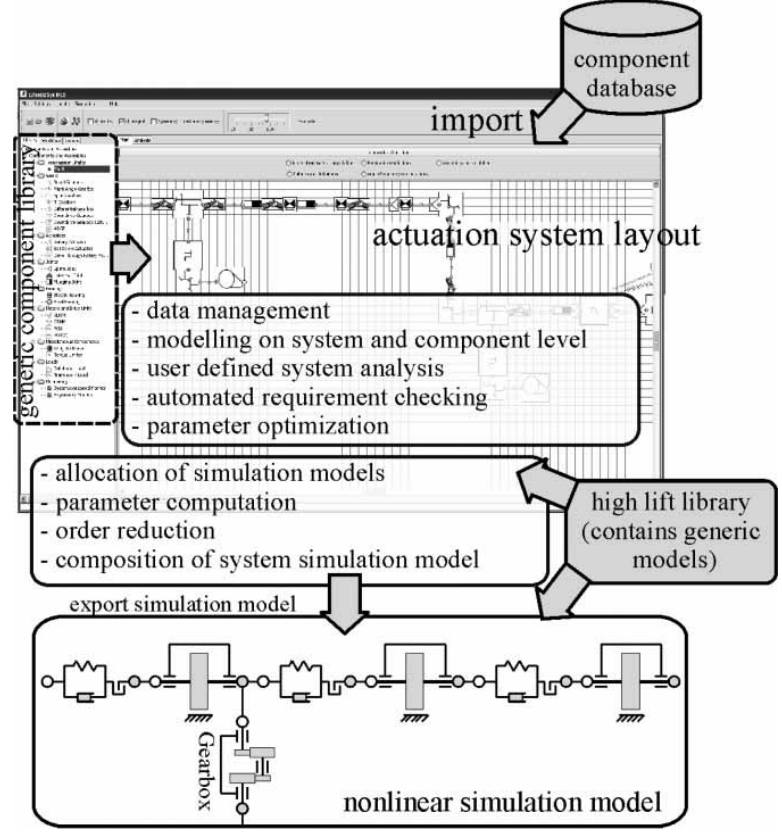

Fig. 3 High lift design assistance tool WissBaSys

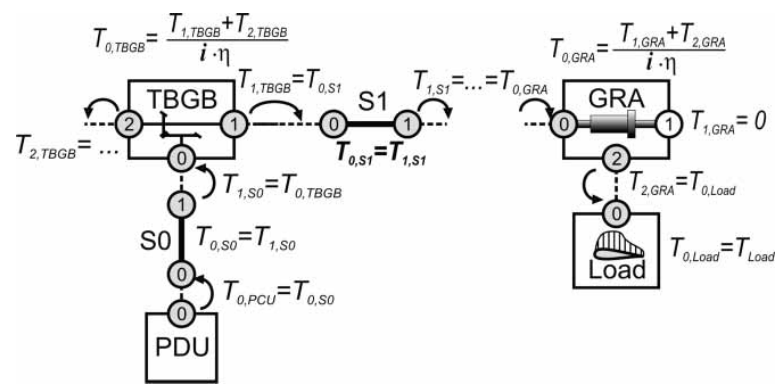

Fig. 4 Recursive procedure determining an architecture-dependent set of equations for the computation of static interface loads

mechanical connections are symbolized by connecting these ports. A set of predefined parameters allows for an automated generation of the system of equations for stationary states. Figure 4 exemplarily illustrates the algorithm for computation of interface loads within the transmission system. While every port features a load variable, $T$, representing the transmitted torque, the equations describing effects on component level have to be specified for all generic components. In this way, computations on system level automatically take changes of the system architecture into account. Besides interface values like torque or static rotational deflection, parameters referring to a specific component drive side, which are inertia and torsional stiffness, can be summed up for adjacent elements and might be transformed to a defined interface position.

After equations and computations for system analysis have been defined, evaluation can be automated. Thus, the effort to perform trade-off studies can be reduced significantly. For a predefined system architecture even parameter optimization becomes available, as described in more detail in reference [3].

The introduced tool offers the possibility to store and give access to different forms of knowledge and experience, which can be used for system design. The following sections present the supported knowledge representation forms and their benefit in the design process. Moreover, information concerning the actuation system's architecture, parameterization, available values, and modelling expertise can be used to assist in the system modelling process. In consequence, the time for model generation can be reduced.

\subsection{Knowledge representation}

Knowledge-based systems are characterized by a strict separation between knowledge representation and knowledge processing [9]. For high lift actuation system design the following forms of knowledge representation are applied.

1. Understanding of system and component functionality is essential. The design task including the system requirements is defined by a set of mathematic expressions. For a specific system architecture, this knowledge is static. Finding a solution implicates application of this knowledge. In WissBaSys, these expressions can be specified on the system and primarily on the component level.

2. Especially in the early concept phase, when many required information are not available, approximations and assumptions based on experience are necessary to start working on the system design. This heuristic proceeding also represents knowledge. In case these heuristics can be expressed in form of mathematic expressions, they can contribute to the set of equations and inequalities defining the system design problem.

3. The external component database, which is presented in Fig. 3 and contains information of existing high lift components, represents experience. This digital memory is now accessible for the user during the design process.

4. Generating complex non-linear simulation models of high lift actuation systems requires expertise in adequate modelling. While generic models can be generated and changed in an external model library, WissBaSys supports automated composition and connection of model elements to a complete system model. Furthermore, parameter computation for generic model blocks is provided.

The knowledge-based design assistance tool WissBaSys provides methods for knowledge processing. The information describing the design problem as well as data representing static experience or heuristic approaches has to be defined by the user. Access 
to and handling of this information are supported. Thus, a strict separation between knowledge representation and processing has been realized. Furthermore, all data are made visible for traceability or adaptation of the design problem.

The core of the problem definition is the system of equations and inequalities expressing restrictions, and mathematical, physical, or heuristic relations between design variables. In order to find a solution, these expressions have to be solved and fulfilled. Whether an acceptable solution can be found depends on the values assigned to the design variables. The following section describes an approach for an automated consistency check including a contradiction of the search space which is based on constraint techniques.

The previous sections outlined the importance of system simulation in the design process. As the system architecture and the assembled components and their parameter values are available as soon as a solution has been found, these information are used to support model generation. Section 4 introduces the concept for an automated model generation.

\subsection{Consistency checking}

The actuation system design task is represented by component and system variables linked to each other by mathematical expressions. As a result, a complex network of equations and inequalities forms during problem definition. All these expressions constrain the solution space. In order to find a valid system design, all variables in this system of equations and inequalities have to be set to a permissible value while all expressions are fulfilled. In other words, a solution has to be consistent. This characteristic is a typical constraint satisfaction problem (CSP). CSPs differ in the types of constraints and the domains of the involved variables. The constraints at hand are algebraic expressions linking two or more variables while real numbers have to be found for the design variables. The range for admissible values can be represented by continuous intervals. In consequence, the design problem can be described as an interval CSP (ICSP).

Application of the ICSP formalization provides the following benefits.

1. Especially mechanical component parameters are often afflicted with tolerances or uncertainties. In early design phases, even more parameters are uncertain or only limited to a margin. Utilization of interval domains allows representation of such uncertainties [10].

2. Constraint propagation is non-directed. Thus, the computational flow is not predefined but depends on the problem at hand. Changes of one of the variable domains are propagated throughout the constraint net, affecting the domains of other variables. Consequently, the search space is reduced significantly although the user may have specified a few variables only.

3. As a side effect of constraint propagation, consistency checking is automated. Thus, system and component requirements are checked within this process and violations are detected.

Constraint propagation is also applicable for underdetermined and incrementally growing inequality systems. WissBaSys applies a kind of Waltz filter algorithm [11] for local tolerance propagation aiming at the so-called hull-consistency [12]. The algorithm presumes undivided interval domains. This assumption is reasonable for the design problem because the physical domains are positive real intervals while the expressions making up the constraint net are mostly continuously differentiable equations. Hence, only the lower and upper bounds need to be taken into account during constraint propagation.

Utilization of local tolerance propagation does not guarantee global consistency; thus, the search space does not necessarily contain a solution even though inconsistency has not been detected. Nonetheless, inconsistency is detected for many configurations. On the other hand, the lacking completeness is outweighed by quick propagation time.

\section{COMPUTER-AIDED MODELLING OF HIGH LIFT ACTUATION SYSTEMS}

The previous sections outline the importance of modelling and simulation within the development process. For example, inertia loads occurring at high speed gradients have to be taken into account for determination of design loads. Numerical simulations favour a reliable prediction of these loads as well as an extensive system analysis. Nonetheless, generation of complex simulation models is a time-intensive task that is often done manually. The presented approach aims at supporting model generation and parameter computation for simulation models in order to enable accurate system analysis in the design process as soon as possible.

\subsection{System modelling}

While the actuation system in total can be divided into the major subsystems PDU, slat flap control computer (SFCC), and shaft transmission system, modelling the mechanical structure of the transmission elements is in focus in the following. Figure 2 exemplarily illustrates the number and types of installed mechanical components. The corresponding abstract model of the branched drive train is a torsional vibrating system. All mechanical elements are characterized by a specific torsional stiffness and a mass moment of inertia. Non-linear effects that have to be taken into account 
are mechanical backlash in transmission gearboxes or at mechanical connections and friction within the components.

Besides these basic characteristics, some elements provide additional features. In order to enable full system functionality, the system model needs to represent gearbox ratios and brake or torque limiter characteristics as well. Consequently, three types of generic model elements are required for modelling the shaft transmission system. These are basically.

1. Rigid elements representing a specific inertia $J$. The inertia's equation of motion

$$
J \cdot \ddot{\varphi}=\sum T_{\text {ext }}-T_{\text {fric }}
$$

depends not only on the specific inertia but also on the external forces $T_{\text {ext }}$ as well as on friction losses $T_{\text {frict }}$ which often depend on the loads and speed [13]. Thus, it seems natural to concentrate component friction in this rigid model.

2. Elastic elements that connect the rigid inertia elements. They take the torsional flexibility and deflection into account. Furthermore, structural damping might be considered in the elastic model block. As a reaction to torsional deflection $\Delta \phi \mathrm{g}$ interface loads

$$
T_{\text {elast }}=c \cdot \Delta \varphi+d \cdot \Delta \varphi
$$

are induced depending on the torsional stiffness $c$ and a damping coefficient $d$. As these elements represent torque transmission, consideration of mechanical backlash $\Delta \phi_{b l}$ within these model elements seems appropriate. Hence, the transmitted torque is

$$
T_{\mathrm{tm}}= \begin{cases}c \cdot\left(\Delta \varphi-\Delta \varphi_{\mathrm{bl}}\right)+d \cdot \Delta \dot{\varphi}: & \Delta \varphi>\Delta \varphi_{\mathrm{bl}} \\ 0: & |\Delta \varphi| \leqslant \Delta \varphi_{\mathrm{bl}} \\ c \cdot\left(\Delta \varphi+\Delta \varphi_{\mathrm{bl}}\right)+d \cdot \Delta \dot{\varphi}: & \Delta \varphi<-\Delta \varphi_{\mathrm{bl}}\end{cases}
$$

3. The third category of model blocks is more diverse and covers all additional functionalities, like (e.g. brake operation). These features can be modelled separately and inserted in the model structure where the respective components demand it.

Figure 5 illustrates the described model structure consisting of rigid and flexible elements. An additional ideal gear reduction stage is inserted in the downdrive branch, thus taking the gear ratio into account.

In order to generate a simulation model that can be used for different tasks, a problem-independent system model is necessary. The object-oriented, equation-based modelling language Modelica $[14,15]$ offers several benefits aiming at automated model generation. Unlike model structures that require the definition of a signal flow, modelling orients on the

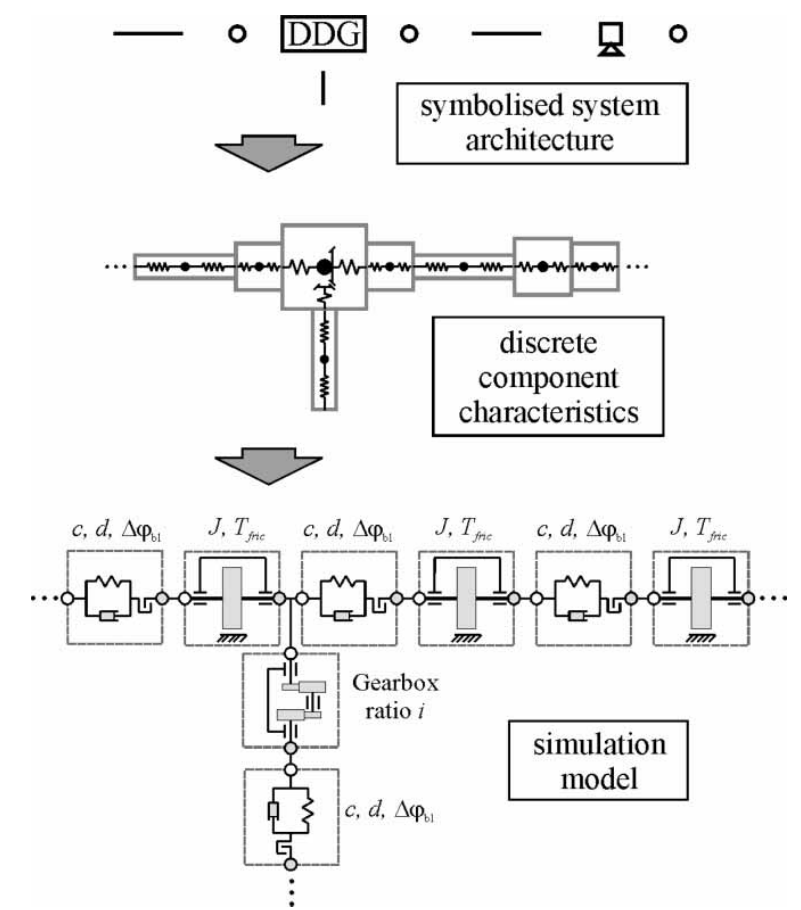

Fig. 5 Extract of a shaft transmission system and related structure of simulation model

physical system structure without the need to determine inputs and outputs explicitly. Models of technical domains like (e.g. mechanics or hydraulics) are connected by special connector elements. These interfaces define potential and flow variables. Potential variables of connected interfaces are required to be equal while the sum of the flow variables is set to zero. While equations for model connection based on the connector elements are generated automatically, equations or differential equations determining the relation between the connector variables have to be defined within the models. Regarding the system architecture described as a non-linear torsional vibrating system, the interface variables defined in the connector elements are an angle $\varphi$ and a transmitted torque $T$. Accordingly, equations (1) and (3) can be implemented directly for modelling.

Supporting system modelling, an extensive and free standard library is available containing mechanical rotational models among others. While an element representing the characteristics of a flexible transmission element considering torsional stiffness, damping, and mechanical backlash is contained in this library, a rigid model taking friction losses into account can be extended based on an ideal inertia element.

Modelica supports two ways of modelling. First, differential equations can be implemented directly, which is helpful for modelling on component level. On the other hand, model composition by combination and connection of predefined generic model blocks via their connectors is the other possibility. Every model code is divided into a section for definition 


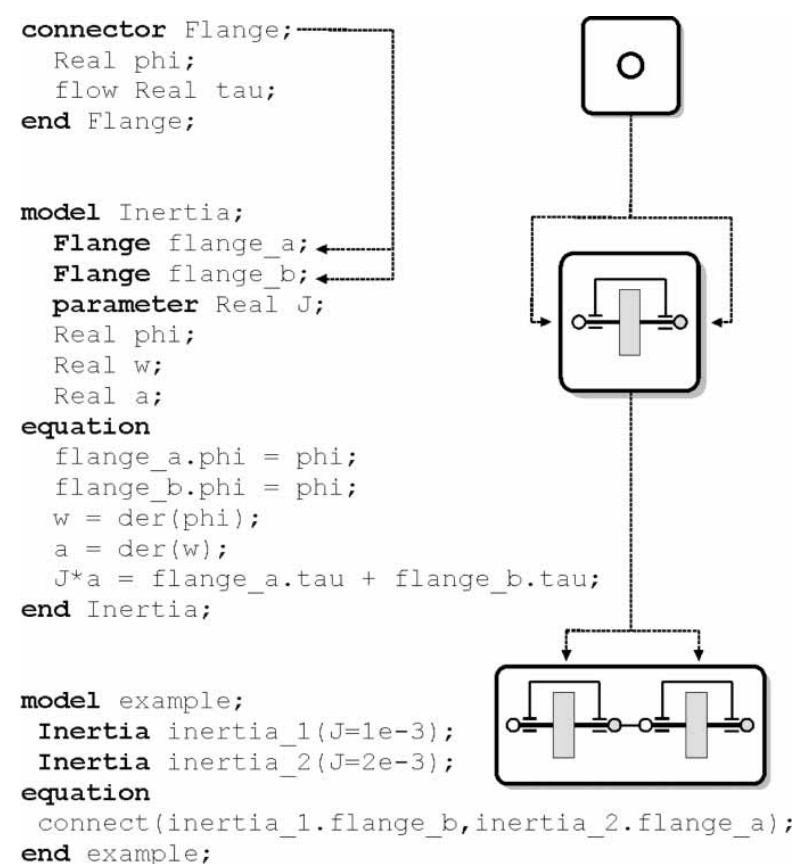

Fig. 6 Example of Modelica code for connectors, subsystems, and systems

of model parameters and specification of submodels. Another part contains the model equations. The equations might be implemented either directly or are expressed by the connection between submodels. Figure 6 demonstrates the code structure exemplarily.

The model structure used in Modelica is helpful for an automated model generation of high lift actuation systems. Detailed modelling expertise can be collected and managed in a generic model library. Thus, modelling on the component level is supported in an appropriate modelling environment which is independent from the design tool WissBaSys. Generic models are defined by their model parameters and the implemented equations. For modelling the shaft transmission architecture, the required knowledge is restricted to the necessary model elements, their parameters as well as their determination, and finally the connection of the submodels. If the system architecture is known and the parametric models as well as the corresponding parameter determination are defined, code generation for the complete system model becomes available. However, the presented computer assistance restricts to model generation while numerical simulation for system analysis can be executed in a powerful external simulation environment.

\subsection{Order reduction}

Owing to the high number of components contained in the shaft transmission system, the resulting simulation model would be of an extreme high order if every element was represented explicitly. Consequently, the simulation time would not be satisfying especially not for real-time simulation. However, a significant lesser level of detail would be sufficient for system analysis. Taking these aspects into account, an order reduction of the actuation system is necessary before the final simulation model is generated.

Most methods available for order reduction of discrete torsional vibrating systems refer to linear systems. Based on discrete values of sectional elements, parameters of a reduced model are determined in a way that allows for an adequate representation of system dynamics. Numerical methods applicable for order reduction of drive trains are presented in reference [16], while another approach using numerical optimization for order reduction with respect to the natural frequencies of a segmented shaft transmission system for flap actuation is described in reference [8].

However, numerical distribution of inertia and stiffness segments suffers a major disadvantage. An explicit allocation of non-linear effects to elements of the reduced system model is not possible. An appropriate distribution of friction effects or mechanical backlash corresponding to the distribution of stiffness and inertia is hardly available.

For this reason, an approach for order reduction based on the system characteristic according to the description in references [17] and [18] is introduced in the following. Long and thin-walled torque shafts connect the PDU to the actuator drive stations in the wing area. Owing to lightweight design and their tube shape, the shaft elements are of minor inertia compared to other transmission elements. On the other hand, downdrive and angle gearboxes are installed within the shaft routing for connecting the branching actuator stations to the main shaft transmission and allow for significant directional changes, respectively. Especially gearboxes represent a high concentration of inertia in the shaft transmission system. Regarding the mass distribution in actuation systems, the creation of lumped inertia elements around components representing a mass concentration seems appropriate.

Figure 7 shows the component inertia of all mechanical components of the Airbus A330/340 flap actuation system. Obviously, gearboxes as well as other components like the system torque limiter or the shaft brake at the wing tip represent high mass concentrations in the drive train. Compared to the surrounding elements, their inertia is dominant. Thus, the generation of lumped inertia by summing up the inertias of the dominant components and its neighbours will lead to a reduced model order representing dominant inertia concentrations only. Order reduction has been achieved according to the following premises.

1. The centre of an inertia concentration is found, if component inertia exceeds that of its direct 


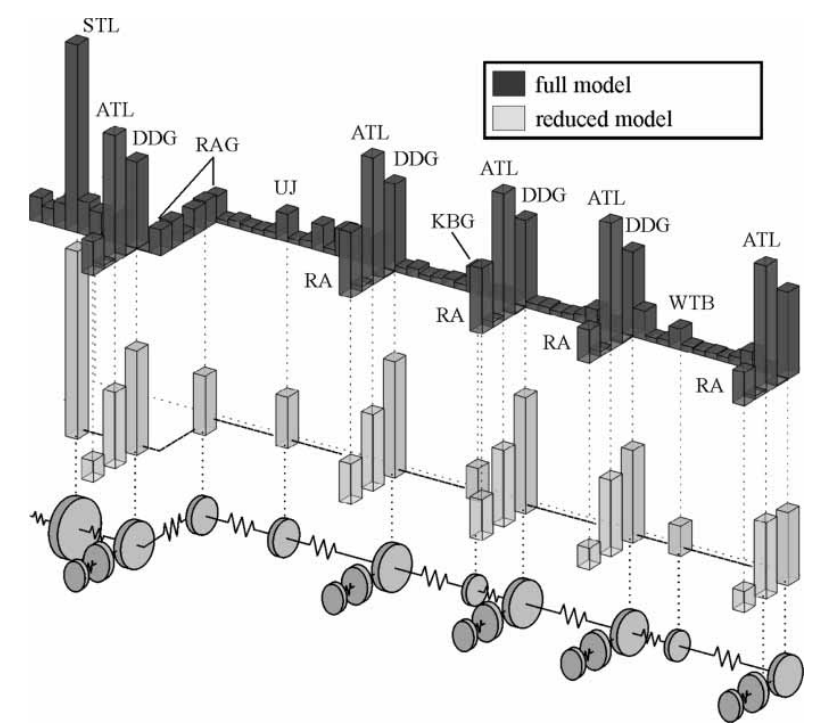

Fig. 7 Inertia distribution in Airbus A330/340 flap actuation system and its reduced model

neighbours significantly. Here a factor of 3 has been used as a threshold.

2. In case neighbouring components feature similar moments of inertia and additionally exceed that of bordering elements, these components also represent an inertia concentration.

In order to allocate and merge elements to lumped inertia blocks, the components between the central elements are shared equally. In case an equal share is not possible and the centre component is a transmission shaft, which does not feature non-linear characteristics, the shaft inertia contributes equally to the neighbouring inertia sections. Otherwise, a component with non-linear characteristics adds to the dominant lumped inertia section.

According to this procedure, precise assignment of components to lumped inertias is guaranteed. Furthermore, respective friction characteristics are bundled in these mass concentrations.

Figure 7 illustrates the resulting lumped inertias of the reduced model. These are connected by the previously introduced flexible elements. The torsional stiffness of these models is derived from the mechanical components connecting the centres of the inertia concentrations. The resulting stiffness values are influenced by components of minor stiffness which are mainly transmission shafts. On the other hand, components that represent high inertia concentrations are generally characterized by high torsional stiffness values.

The natural frequencies of a model characterize its system dynamics. The following comparison of the linear full-scale and reduced model allows for an evaluation of the effects on the system's dynamics resulting from order reduction according to this approach.

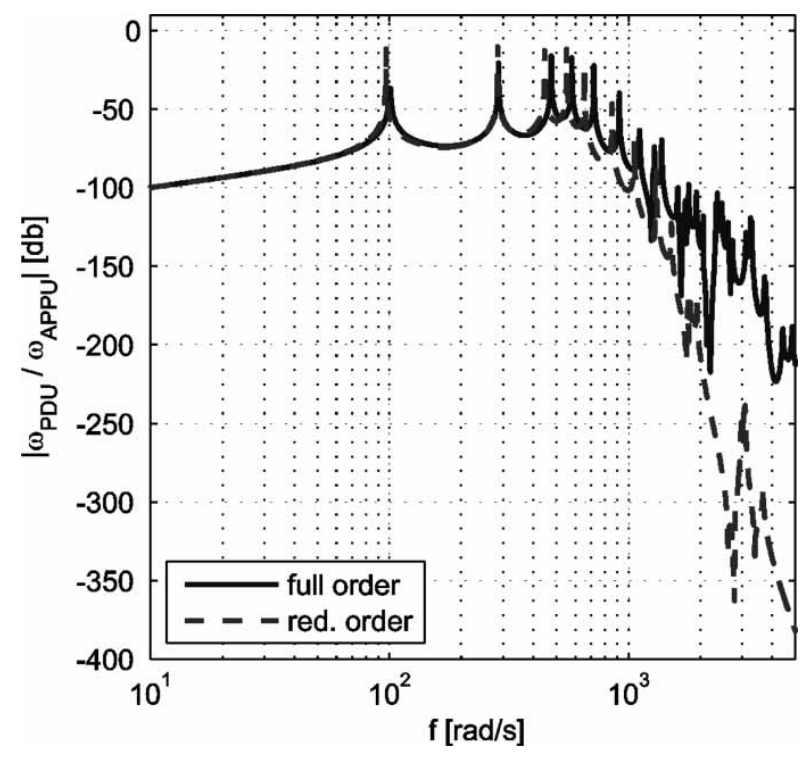

Fig. 8 Frequency response for full-scale and reduced model of Airbus A330/340 shaft transmission system

Regarding the multi-degrees-of-freedom mass-spring vibrating system the equations of motion in vector form are

$$
\mathbf{M} \cdot \ddot{\boldsymbol{y}}(t)+\mathbf{K} \cdot \boldsymbol{y}(t)=\boldsymbol{h}(t)
$$

where $\boldsymbol{y}=\left[\varphi_{1} \ldots \varphi_{f}\right]^{\mathrm{T}}$ represents the $f \times 1$ position vector of the lumped inertias' rotational degrees of freedom. The diagonal matrix $\mathbf{M}$ contains the inertia values, while the symmetric matrix $\mathbf{K}$ represents the stiffness components [19]. The $f \times 1$ vector $\boldsymbol{h}$ represents external forces. Using the state vector $\boldsymbol{x}(t)=$ $\left[\begin{array}{ll}\boldsymbol{y}(t)^{\mathrm{T}} & \dot{\boldsymbol{y}}(t)^{\mathrm{T}}\end{array}\right]^{\mathrm{T}}$, equation (4) can be expressed by the state equation

$$
\dot{\boldsymbol{x}}(t)=\mathbf{A x}(t)+\boldsymbol{b}(t)
$$

with the system matrix

$$
\mathbf{A}=\left[\begin{array}{cc}
\mathbf{0} & \mathbf{E} \\
-\mathbf{M}^{-1} \mathbf{K} & \mathbf{0}
\end{array}\right]
$$

The system matrix enables determination of the natural frequencies and the frequency responses. Figure 8 compares the amplitude frequency response at the wing tip for an excitation at the PDU output. As illustrated, the frequency responses of full and reduced model show very good conformity for a wide frequency spectrum. Especially the lower natural frequencies are almost identical. Thus, dynamic similarity in the relevant frequencies has been achieved. While the lower frequencies are most important for system dynamics, the applied order reduction proves appropriate. 


\subsection{Computer-aided model generation}

The previous sections introduced the model structure for simulation of high lift actuation systems and an approach for order reduction. This can be used for an automated model generation which is presented in the following. The major components used for modelling the transmission system are stiff lumped inertia models including friction losses and flexible spring-damper elements with mechanical backlash. Specialized models represent functions that can be allocated to a specific component.

While modelling expertise is created and managed externally in the form of a specialized component library using the modelling language Modelica, order reduction of the shaft transmission system, parameter computation for generic submodels, and assembling these submodels to a system model can be automated. The supported computational assistance is based on an adequate model library. Especially those elements representing the stiff and elastic elements are needed to enable modelling the transmission system. First, the respective models have to be defined in WissBaSys while the corresponding model parameters are read out automatically. Afterwards, the computation of these parameters has to be specified for the calculation of results from serial component values. The definition of models and the computation of their parameter sets can then be applied to segments of the shaft transmission.

In contrast to the required models for representation of the vibrating system, specific models have to be defined within the respective components. Moreover, the references between component and model parameters need to be determined. Thus, special models can be inserted in the transmission model at the corresponding position.

The final step for modelling the actuation system is the order reduction of the shaft transmission. Sections that merge to lumped inertia or stiffness elements can be proposed according to the previously presented procedure. A visualization of the section borders allows for a graphical modification by the user.

Thus, determination of the necessary types and numbers of model elements, their interconnection, and the values of their parameters is available. This information can easily be expressed in Modelica code.

This approach offers an automated model generation as well as a transparent and traceable visualization of the order reduction and parameter computation for dedicated submodels, as illustrated in Fig. 9. Furthermore, model adaptation due to the changes of component parameters or even the system architecture can be handled. In this way, not only a significant time reduction for modelling can be achieved, but the transparency of the process is enhanced too. At the same time, modelling expertise is accessible and editable by the user in a practical modelling and

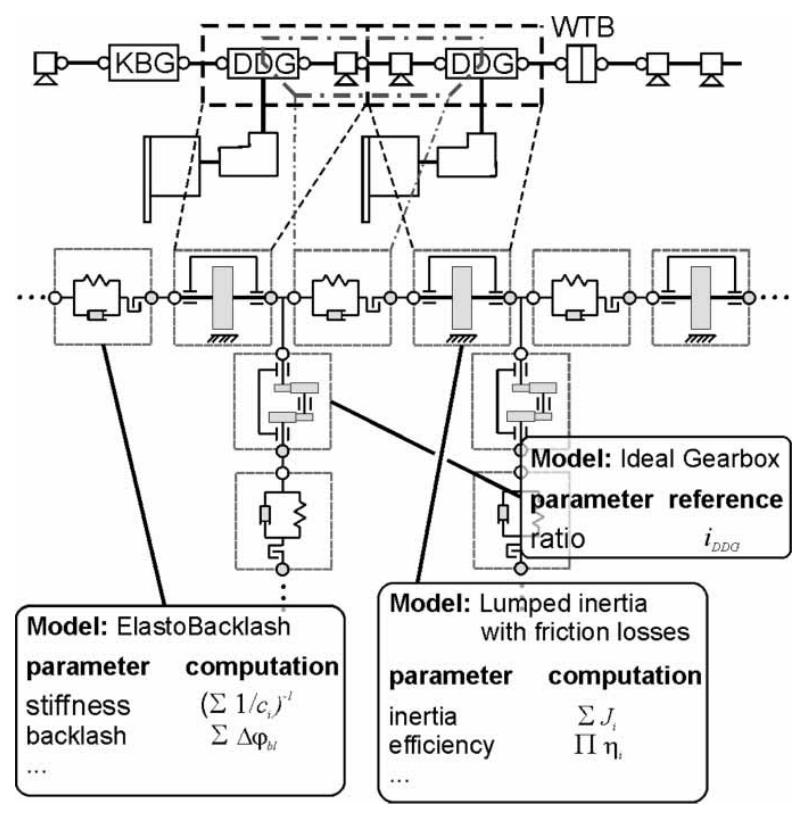

Fig. 9 Concept for an automated model generation and correlation between components and model elements

simulation environment, which might also take over the numerical system simulation and analysis.

\section{CONCLUSIONS}

Numerical simulation is of significant importance for high lift actuation system design, analysis, and verification. However, extensive simulation is hardly available in preliminary design. This fact owes to missing and varying component data as well as an enormous effort for modelling and model adaptation, which is often executed manually. Moreover, specialized and integrated computer assistance supporting high lift actuation design does not exist. The objective of the research program WissBaSys is to provide a tool offering extensive, knowledge-based assistance for system design.

The approach presented in this article enables knowledge representation by algebraic expressions incrementally forming a constraint network. The application of constraint techniques allow for an automated consistency checking as well as a contradiction of the solution space which can be extremely helpful in preliminary design. Furthermore, the effects of structural changes of the system architecture on the design problem are minimized, because design relevant equations are basically defined on the component level. Hence, the effort for redefinition of the design problem is reduced significantly.

In order to support system development beyond conceptual design, transferring available data concerning the actuation system architecture and com- 
ponent parameter values to a complex non-linear simulation model can be automated. While modelling expertise is created and managed in a specialized modelling environment, the composition of specified models to a complex system model as well as the computation of the required model parameters can be automated. Furthermore, an approach for the generation of a reduced model of the shaft transmission system consisting of numerous components is introduced. Taking advantage of the transmission system characteristic, an order reduction of the Airbus A330/340 flap actuation system demonstrates that a dynamic similarity of the reduced model can be achieved. Based on the presented rules for order reduction, an automated proposal for a reduced model can be realized. The multi-disciplinary modelling language Modelica favours an automated transformation of available system and component data to a simulation-ready system model.

\section{ACKNOWLEDGEMENT}

The authors thank Airbus Operations GmbH for promoting the investigations of the research project WissBaSys.

(C) Authors 2011

\section{REFERENCES}

1 Recksiek, M. Advanced high lift system architecture with distributed electrical flap actuation. In Proceedings of the Workshop on Aircraft system technologies, Hamburg, 2009.

2 Rudolph, P. K. C. High-lift systems on commercial subsonic airliners. NASA Contractor Report no. 4746, National Aeronautics and Space Administration, Ames Research Center, Moffett Field, CA, USA 1996.

3 Pfennig, M., Carl, U. B., and Thielecke, F. Recent advances towards an integrated and optimized design of high lift actuation systems. In Proceedings of the SAE 2009 AeroTech Congress \& Exhibition, Seattle, 2009.

4 Fielding, J. P. Introduction to aircraft design, 1999 (Cambridge University Press, Cambridge).

5 Jackson, S. Systems engineering for commercial aircraft, 1997 (Ashgate Publishing, Singapore).

6 Howe, D. Aircraft conceptual design synthesis, 2000 (Professional Engineering Publishing, St Edmunds, London).

7 Pfennig, M. and Thielecke, F. Konzepte und Methoden für eine rechnergestützte Auslegung der Antriebssysteme von Hochauftriebssystemen. In Deutscher Luft und Raumfahrt-kongress, Darmstadt, 2008.

8 Neumann, U., Holert, B., and Carl, U. Für eine sichere Landung - Simulation von Landeklappenantriebssystemen. antriebstechnik, Band, 2004, 43(4), 70-74.

9 Beierle, C. and Kern-Isberner, G. Methoden wissensbasierter Systeme, 2000 (Vieweg Verlag, Braunschweig).
10 Hyvonen, E. Constraint reasoning based on interval arithmetic. In Proceedings of the 11th International Joint Conference on Artificial intelligence, Detroit, 1989

11 Davis, E. Constraint propagation with interval labels. Artif. Intell., 1987, 32, 281-331.

12 Lhomme, 0. Consistency Techniques for Numeric CSPs. In Proceedings of the 13th International Joint Conference on Artificial intelligence, Chambéry, 1993, pp. 232-238.

13 Pelchen, C., Schweiger, C., and Otter, M. Modeling and simulating the efficiency of gearboxes and of planetary gearboxes. In Proceedings of the 2nd International Modelica Conference, Oberpfaffenhofen, March 2002, pp. 257-266.

14 Modelica Association (Ed.) ModelicaTM - A unified object-oriented language for physical systems modeling. Tutorial, Version 1.4, 15 December 2000.

15 Tiller, M. M. Introduction to physical modelling with modelica, 2004 (Kluwer Academic Publishers, Boston).

16 Laschet, A. Simulation von Antriebssystemen, 1988 (Springer Verlag, Berlin).

17 Ruprecht, T., Thielecke, F., and Recksiek, M. SIVA - a testrig for the validation of high lift component models. In Proceedings of the 1st International Workshop on Aircraft system technologies, Hamburg, 29-30 March 2007.

18 Rechter, H. and Richter, M. Die Simulation als Hilfsmittel bei der Entwicklung und Integration der A330/340Hochauftriebs-systeme. Deutscher Luft- und Raumfahrtkongress Band I, Göttingen, 1993.

19 Müller, P. C. and Schiehlen, W. O. Linear vibrations, 1985 (Kluwer Academic Publishers, Lancaster).

\section{APPENDIX}

\section{Notation}

b

$c$

$d$

E

$f$

$\boldsymbol{h}$

J

K

$t$

$T$

$\boldsymbol{x}$

$y$

$\varphi$

system matrix

input vector

torsional stiffness

damping

identity matrix

degrees of freedom

excitation vector

moment of inertia

stiffness matrix

inertia matrix

time

torque

state vector

position vector

angle

\section{Abbreviations}

ATL actuator torque limiter

DDG downdrive gearbox

RA rotary actuator

RAG right-angle gearbox

STL system torque limiter 